\title{
Analysis of Explosives by GC-UV
}

J an Andrasko, Ludmila Lagesson-Andrasko, J ohan Dahlén and Bengt-Harald Jonsson

The self-archived version of this journal article is available at Linköping University Institutional Repository (DiVA):

http:// urn.kb.se/ resolve?urn=urn:nbn:se:liu:diva-139621

N.B.: When citing this work, cite the original publication.

Andrasko, J ., Lagesson-Andrasko, L., Dahlén, J., J onsson, B., (2017), Analysis of Explosives by GC-UV, J ournal of Forensic Sciences, 62(4), 1022-1027. https:// doi.org/ 10.1111/1556-4029.13364

Original publication available at:

https:/ doi.org/ 10.1111/ 1556-4029.13364

Copyright: ASTM International / Wiley: 12 months

http:// eu.wiley.com/WileyCDA/ 
Jan Andrasko ${ }^{1}$, Ludmila Lagesson-Andrasko ${ }^{1}$, Johan Dahlén ${ }^{2}$, Bengt-Harald Jonsson ${ }^{2}$

${ }^{1}$ GC-UV center, Kobergsgränd 2, SE-587 31 Linköping, Sweden

${ }^{2}$ Department of Physics, Chemistry and Biology (IFM), University of Linköping, SE-181 83 Linköping, Sweden

\section{Analysis of Explosives by GC-UV Method}

ABSTRACT: A mixture of explosives was analysed by gas chromatography (GC) linked to ultraviolet (UV) spectrophotometry that enabled detection in the range of $178-330 \mathrm{~nm}$. The gas phase UV spectra of 2,4,6-trinitrotoluene (TNT), 2,4-dinitrotoluene (DNT), ethylene glycol dinitrate (EGDN), glycerine trinitrate (NG, nitroglycerine), triacetone triperoxide (TATP), pentaerytritol tetranitrate (PETN) were successfully recorded.

The most interesting aspect of the current application is that it enabled simultaneous detection of both the target analyte and its decomposition products. At suitable elevated temperatures of the transfer line between the GC instrument and the UV detector, a partial decomposition was accomplished. Detection was made in real time and resulted in overlaid spectra of the mother compound and its decomposition product. Hence, the presented approach added another level to the qualitative identification of the explosives in comparison to traditional methods that relies only on the detection of the target analyte. As expected, the decomposition product of EGDN, NG and PETN was NO, while TATP degraded to acetone. DNT and TNT did not exhibit any decomposition at the temperatures used.

UV detection in gas phase is a sensitive and selective universal detection that is suitable for identification and quantification. It is robust with easy maintenance, no moving parts, no ionization and no vacuum pumps. It is particularly useful for detection and identification of isomers.

KEYWORDS: Forensic science, ultraviolet detection, gas chromatography - ultraviolet spectrophotometry, ultraviolet spectra, explosive analysis, triacetone triperoxide, nitrate esters 
The detection of explosives and related compounds is an issue of major importance in different fields. Nearly all known analytical methods have already been investigated for their applicability to analyze explosives (1-18). A review of the instrumentation used for trace detection of explosives was given by Moore in 2004 (1) and was supplemented with advances in 2007 (2). Some more recent reviews of the various analytical methods can be found in the literature (3-18). In general, gas chromatography (GC) is the method of choice, while a range of different detectors can be coupled to this instrument.

The usual GC systems cannot be applied to explosives without slight modifications of temperature settings as all explosives are not stable at higher temperatures. The deflagration temperatures of some common explosives can be found in literature (19) and are in many cases as low as about $200^{\circ} \mathrm{C}$. At such elevated temperatures, a sudden decomposition of explosives may take place. For example, the nitrate esters such as ethylene glycol dinitrate (EGDN), nitroglycerine (NG) and pentaerythritol tetranitrate (PETN) decompose rapidly, whereas nitroaromatics such as dinitrotoluene (DNT) and trinitrotoluene (TNT) are more stable.

Gas chromatography with ultraviolet detection (GC-UV) is a hyphenation of gas chromatography and UV spectrophotometry in gas phase. The instrument INSCAN model 175 , developed and built by Verner Lagesson and Ludmila Lagesson-Andrasko, was successfully employed in several studies $(20,21)$. A spectral library containing more than 1400 gas phase UV spectra, as a basis for identification of compounds and determination of specific functional groups, was established.

One of the GC-UV method advantages is an illustrative three dimensional presentation of the chromatogram. It is displayed at real time and the decomposition process can be followed visually on line. With benefit, this method can be employed in parallel with GC-MS for verification and as its complement. The simplicity and robustness of GC-UV is another advantage. It can easily be modified to a mobile design for use in the field or for continuous monitoring of industrial processes online. The GC-UV is excellent for identification of isomers and small gaseous molecules. On comparison with GC-IR detection, the sensitivity of GC-UV can be as much as 1000 times higher, depending on the 
absorption properties of electronic spectra. The UV spectra in vapor phase are well defined because they are not influenced by solvent effects. Many substances show also vibrational fine structure overlaid to absorption bands. These properties make the method particularly suitable for a reliable identification of unknown compounds. Compounds belonging to a certain class (i.e. aldehydes) are characterized by similarities in their UV spectra, which can be useful in classification of unknowns. The aim of this study was to obtain UV spectra in gas phase for some common volatile explosives. In the applied approach, the temperature of the transfer line between the GC and the UV detector was optimized to induce partial decomposition in order to enable simultaneous detection of the mother compound and its decomposition products.

\section{Materials and Methods}

\section{Instrumentation}

GC-UV analyses of the explosives were performed using an HP 5890 GC (Agilent technologies) connected to an ultraviolet spectrophotometer INSCAN model 175 ( GC-UV center, Linköping, Sweden), described earlier $(20,21)$. The spectrograph, with a focal length of $120 \mathrm{~mm}$ with interchangeable grating and a variable inlet slit, was linked to a 1024 element photo diode array detector. A deuterium lamp, model L 2196 (Hamamatsu) was employed. During the analysis, UV spectra (178-330 nm) were recorded every third second. The intervals for sampling of UV spectra can freely be chosen. During these intervals, the spectra are continuously accumulated (e.g. 12 accumulations in three seconds intervals used here) and the results recorded at the end of each interval. Thus all narrow peaks are also detected and recorded.

The acquisition of data was carried out using Instaspec (Orion Instruments, Stratford, CT, USA) software. The raw data were transferred to GRAMS AI (Galactic Industries Corporation, Salem, New Hampshire, USA) for three dimensional presentation, evaluation and extraction of UV spectra. 
The GC separation columns used were an Ultra 2 (Agilent technologies; $25 \mathrm{~m} \times 0.2 \mathrm{~mm} \times 0.33 \mu \mathrm{m}$ ) for the analysis of the more volatile explosives and a HP 5 (Agilent technologies; $15 \mathrm{~m} \times 0.25 \mathrm{~mm} \times 0.25$ $\mu \mathrm{m})$ column for the analysis of PETN. The temperature program used (unless otherwise stated) started at $60^{\circ} \mathrm{C}$ (held for $1 \mathrm{~min}$ ) and the temperature was thereafter increased by $10^{\circ} \mathrm{C} /$ minute until the final temperature was reached. The final temperatures were varied in a series of experiments to evaluate the decomposition process. The carrier gas was nitrogen and the linear flow was kept to about $30 \mathrm{~cm} / \mathrm{s}$ (at $60^{\circ} \mathrm{C}$ ) for both analytical columns. The transfer line connecting the $\mathrm{GC}$ chromatograph to the flow cell of the spectrophotometer was $100 \mathrm{~cm}$ long and was kept at different programmable temperatures $\left(150-240^{\circ} \mathrm{C}\right)$. The volume of the gas flow cell was $170 \mu$, the length 80 $\mathrm{mm}$, the temperature $200^{\circ} \mathrm{C}$ and the flow through it enhanced using nitrogen gas at $12 \mathrm{ml} / \mathrm{min}$.

Samples

Samples of various explosive compounds were a gift from NFC (National Forensic Centre, Linköping, Sweden). The samples of EGDN, NG, 2,4-DNT and TNT were obtained as a mixture in acetone, in which each substance had a concentration of $20 \mathrm{ng} / \mu \mathrm{l}$. Generally, $1 \mu \mathrm{l}$ aliquots were injected splitless. The samples of TATP (triacetone triperoxide) and PETN were used as pure powders that were introduced onto the column using microthermal desorption (23). Names, abbreviation and chemical structures of the explosives included in the study are summarized in Table 1.

\section{Results}

Analysis of EGDN, NG, DNT and TNT

A mixture of EGDN, NG, DNT and TNT (each $20 \mathrm{ng} / \mu \mathrm{l}$ ) in acetone was used as the reference solution that was analyzed by the GC-UV system. To study the temperature dependent decomposition of the explosives, the temperature of the transfer line connecting the GC to the flow cell of the UV 
spectrophotometer was varied. The temperature program, in these series of experiments, started at $40^{\circ} \mathrm{C}$ (held for $1 \mathrm{~min}$ ) and the oven temperature was thereafter increased by $10^{\circ} \mathrm{C} / \mathrm{min}$ until the final temperature was reached. The final oven temperature was always the same as the temperature of the transfer line.

The UV spectra obtained for the nitroaromatics (DNT, TNT) were unaffected by the different transfer line temperatures tested $\left(150,170,200\right.$ and $\left.240^{\circ} \mathrm{C}\right)$. Recorded gas phase spectra of the two compounds, obtained using a transfer line temperature of $240^{\circ} \mathrm{C}$, are presented in Fig. 1.

On the contrary, the appearance of spectra of EGDN and NG were influenced by the temperature of the transfer line. At a transfer line temperature of $150^{\circ} \mathrm{C}$ no decomposition could be detected. A part of a three-dimensional chromatogram recorded at these conditions are shown in Fig. 2 . The presence of dinitroglycerines (glycerine dinitrate) can be noted in this figure; these compounds eluted slightly before NG. The wavelengths of maximum absorption of EGDN and NG were found to be $186.9 \mathrm{~nm}$ and 186.4, respectively. The pure UV spectra of EGDN and NG, which were quite similar, are shown in Fig. 3.

The retention times of EGDN and NG, exhibiting higher vapor pressures, were constant and rather short. They were not influenced by the temperature of the transfer line.

When the temperature of the transfer line was increased, the peaks for EGDN and NG started to decompose to nitric oxide (NO) and the observed spectra were a combination of pure spectra of the mother compound and the spectrum of NO. Hence, the retention times of mother substance and its decomposition products were the same, which means that the decomposition takes place in the transfer line, and not on the analytical column during the chromatographic separation. In Fig. 4, a three-dimensional chromatogram, recorded at a transfer line temperature of $220^{\circ} \mathrm{C}$, exemplify the degradation of NG to NO. Extracted UV spectra of the NG peak at four different transfer line 
temperatures are illustrated in Fig. 5. As can be seen, the degree of decomposition increased with the transfer line temperature. For comparison, the UV spectrum of pure NO is shown. This pure NO sample was prepared in the laboratory and its spectrum obtained using the same analytical conditions as were used for the explosives. Also dinitroglycerine and EGDN, having shorter retention time than NG, decompose to NO in similar manner as NG. As can be seen in Fig. 5, the decomposition of NG to NO is almost complete at the highest temperature employed $\left(240^{\circ} \mathrm{C}\right)$. At temperatures between $180^{\circ} \mathrm{C}$ and $200^{\circ} \mathrm{C}$, there was only a partial decomposition of NG to NO, which means that both species could be detected. Such conditions offers three aspects of NG identification: the retention time of NG, the UV spectrum of NG and the spectrum of the decomposition product (NO) overlaid onto the spectrum of NG. Therefore, keeping the temperature of the transfer line at 180 $200^{\circ} \mathrm{C}$ provides the best condition for the identification of explosive nitrate esters by GC-UV. It is known that explosive nitrate esters decompose thermally to several substances, i.e. $\mathrm{NO}, \mathrm{N}_{2} \mathrm{O}$, $\mathrm{CO}, \mathrm{CO}_{2}, \mathrm{HONO}(24)$. In this study, NO was found to be dominating the obtained UV spectra.

\section{Analysis of TATP}

TATP is a peroxide type of explosive and cannot be detected by nitrogen specific chemiluminescence detectors such as thermal energy analyzer (TEA). The analyses of TATP were performed by microthermal desorption (23) of pure substance. The reason for not using a solution of TATP was to obtain the best information about the decomposition of this very unstable compound. Moreover, TATP is commonly found as a powder or in post explosion residues. Microscopic particles were attached to the inner wire of the SPME holder with the fiber removed, after which the holder with sample was introduced to the GC injector. The oven temperature program of the $\mathrm{GC}$ started at $60^{\circ} \mathrm{C}$ (held for $1 \mathrm{~min}$ ), and was thereafter increased by $10^{\circ} \mathrm{C} /$ minute until a final temperature of $160^{\circ} \mathrm{C}$ was reached. In these experiments, the transfer line temperature was varied between $160^{\circ} \mathrm{C}$ and $240^{\circ} \mathrm{C}$. 
Fig. 6 shows the three-dimensional chromatogram recorded using a transfer line temperature of $180^{\circ} \mathrm{C}$. Three peaks were observed in this chromatogram. The air peak caused by the sample introduction was followed by a peak identified as acetone (spectrum of acetone is for comparison shown in Fig. 7) and the TATP peak. The wavelength of maximum absorption $\left(\lambda_{\max }\right)$ of TATP could not be detected within the UV range $(178-330 \mathrm{~nm})$ enabled by the employed instrumentation; $\lambda_{\max }$ of TATP wavelength is shorter than $178 \mathrm{~nm}$. The presence of acetone in the TATP sample may have two different origins. TATP may decompose to acetone on storage and the formed acetone be adsorbed to the TATP powder. However, the most probable explanation for this observation is that the acetone in the sample is a residual solvent from the preparation of TATP.

Fig. 7 shows the UV spectrum of the TATP peak using different transfer line temperatures. As can be seen, the spectrum of pure TATP was overlaid by the spectrum of acetone, which is due to decomposition of TATP to acetone in the transfer line. The decomposition of TATP, and consequently also the formation of acetone, increased with the transfer line temperature, although a slight decomposition could be observed already at the lowest temperature tested $\left(160^{\circ} \mathrm{C}\right)$. For qualitative analysis of TATP, a transfer line temperature in the range $180-200^{\circ} \mathrm{C}$ would allow for simultaneous detection of TATP and its decomposition product (acetone) at the same retention time, which would improve the reliability of the identification.

If desired, a pure UV spectrum of TATP can be obtained by subtracting the spectrum of pure acetone from the spectrum observed.

Analysis of PETN

PETN is a nitrate ester explosive with lower vapor pressure as compared to EGDN and NG. To monitor its decomposition, another, shorter GC capillary column (HP 5, 15m) was used. The oven temperature program started at an initial temperature of $60^{\circ} \mathrm{C}$ (held for $1 \mathrm{~min}$ ) and was thereafter 
increased by $10^{\circ} \mathrm{C} / \mathrm{min}$ until a final temperature of $180^{\circ} \mathrm{C}$ was reached. The temperature of the transfer line was varied between $180^{\circ} \mathrm{C}$ and $240^{\circ} \mathrm{C}$. Sample introduction was made by microthermal desorption of pure powdered PETN. The UV spectrum of PETN was obtained and its partial decomposition to NO observed. This decomposition was more pronounced at higher temperatures of the transfer line (data not shown) in a manner similar to the decomposition of other nitrate esters. Fig. 8 depicts the UV spectra of PETN recorded at the transfer line temperature of $180^{\circ} \mathrm{C}$ and of pure PETN after subtraction of NO. In addition to its decomposition to NO, other peaks in the resulting chromatograms were detected. Such peaks are known e.g. from analysis by GC-TEA [19] and their nature is not known. They can represent decomposition products of PETN, but may also be impurities. In our experiments, an intense peak eluting before PETN decomposed to NO at elevated temperatures. This indicates that this unknown peak is also nitrate ester. As in the analyses of TATP, a peak identified as acetone was detected in the PETN samples. It cannot be a decomposition product of PETN and is presumably adsorbed residue of solvent used in the preparation of the explosive.

Sensitivity

The sensitivity of detection by GC-UV is depending on the absorption properties of the analyzed compound. For the GC-UV instrumentation employed in this study, the LOD (limit of detection) was in the order of $1 \mathrm{ng}$ of injected explosives. This sensitivity can be improved e.g. by using CCD detection instead of diode array detection. The main advantage of the GC-UV detection is not the highest sensitivity, but the correct and reliable identification of the analyzed explosives and their degradation products. 


\section{Discussion and Conclusion}

In modern chemical analyses the identification of the compounds detected must fulfill several criteria. Thus, for example, in forensic analysis of explosives, the results of an initial analytical method should be confirmed by another, independent method. For a single analysis by gas chromatography combined with a suitable detector, the detected compound is characterized by its retention time together with selectivity of the detection or a combination of retention time and spectrum of the eluted chromatographic peak. An example of the first approach is chemiluminescence detection of NO formed by a pyrolysis of the compound by GC-TEA or explosive sniffer analysis. Example of the second approach is GC-MS analysis. The combination of GC-TEA or explosive sniffer analysis and of GC-MS analysis represents a common approach to achieve a reliable identification of detected compounds.

In this study, qualitative GC-UV analysis of a number of explosives was successfully performed. The main advantage of the developed method is that it fulfills three identification criteria in one single analysis. In addition to the retention time and UV spectra of the explosive compounds, their decomposition products were also observed and identified. This was made possible by using suitable temperatures of the transfer line where the formation of decomposition products occurred. It is obvious that the decomposition of the explosives occurs in the transfer line and not already in the injector or on the separation column. These decomposition products (NO, acetone) are co-eluted with their mother compound and their UV spectra and the spectra of the mother compound were overlaid at the same retention time. Their formation is strongly influenced by the choice of the temperature of the transfer line (see e.g. Fig. 5) when all the other system temperature settings are kept constant.

Thus EGDN, dinitroglycerine, NG, PETN and their decomposition product NO co-eluted and their spectra were overlaid. In a similar way, TATP and its decomposition product acetone co-eluted and their UV spectra superposed each other. A partial decomposition of the mother compound was 
aimed at as this allows for simultaneous detection of the explosive compound and its decomposition products. In this context, optimum transfer line temperatures were in the range of $180-200^{\circ} \mathrm{C}$. Hence, at this temperature both the spectrum of the pure explosive and its degradation products (NO, acetone) could be obtained.

The presented investigation shows GC-UV methods advantages for the analysis of explosives. Its reliability and visual presentation of the results on decomposition products. The aim of this study was not to present the GC-UV method as a method which is superior to the other methods such as GC-MS. The GC-UV method should be considered as an alternative method and as a complement to these methods for analysis of explosives. The GC-UV method is non-destructive to the sample analyzed and may easily be combined with e.g. GC-MS connected in series.

Our instrument employs a limited range of UV. The use of a vacuum ultraviolet detector operating at UV range $120-240 \mathrm{~nm}$ (22) would further improve these results. This should be a next step in future development of the application of the GC-UV method as a powerful tool in analytical chemistry.

\section{Acknowledgements}

The authors are grateful to the National Forensic Centre in Linköping for providing samples of explosives. 


\section{References}

1. Moore DS. Instrumentation for trace detection of high explosives. Rev Sci Instr 2004;75:2499-2512.

2. Moore DS. Recent advances in trace explosive detection instrumentation. Sens Imaging 2007;8:9-

38.

3. Barron L, Gilchrist E. Ion chromatography-mass spectrometry. A review of recent technologies and applications in forensic and environmental explosive analysis. Anal Chim Acta 2014;806:27-54.

4. Johns C, Hutchinson JP, Guit RM, Hilder EF, Haddad PR, Macka M, Nestertenko PN, Gaudry AJ, Docinoski GW, Breadmore MC. Micellar electrokinetic chromatography of organic and peroxidebased explosives. Anal Chim Acta 2015;876:91-97.

5. López-López M, García-Ruiz C. Infrared and Raman spectroscopy techniques applied to identification of explosives. Trends Anal Chem 2014;54:36-44.

6. Krawczyk T, Baj S. Review: Advances in the determination of peroxides by optical and spectroscopic methods. Anal Letters 2014;47:2129-2147.

7. Yan QL, Zeman S, Elbeigh A. Recent advances in thermal analysis and stability evaluation of insensitive plastic bonded explosives (PBXs). Termochim Acta 2012;537:1-12.

8. Willer U, Schade W. Photonic sensor devices for explosive detection. Anal Bioanal Chem 2009;395:275-282.

9. Gottfried JL, DeLucia Jr FC, Munson CA, Miziolek AM. Laser-induced breakdown spectroscopy for detection of explosive residues: a review of recent advances, challenges and future prospects. Anal Bioanal Chem 2009;395:283-300.

10. Leahy-Hoppa MR, Fitch MJ, Osiander R. Tetrahertz spectroscopy techniques for explosive detection. Anal Bioanal Chem 2009;395;247-257.

11. Romolo FS, Cassioli L, Grossi S, Cinelli G, Russo MV. Surface sampling and analysis of TATP by swabbing and gas chromatography/mass spectrometry. Forens Sci Int 2013;224:96-100. 
12. Burks RM, Hage DS. Current trends in the detection of peroxide-based explosives. Anal Bioanal Chem 2009;395:301-313.

13. Zuck A, Greenblatt J, Zifman A, Zaltsman A, Kendler S, Meltzer S, I. Fisher I. Explosive detection by microthermal analysis. J Energ Mat 2008;2613:163-180.

14. Pumera M. Trends in analysis of explosives by microchip electrophoresis and conventional CE. Electrophoresis 2008;29:269-273.

15. Gaurav D, Malik AK, Rai PK. High-performance liquid chromatographic methods for the analysis of explosives. Crit Rev Anal Chem 2007;37:227-268.

16. Gaurav D, Kaur V, Kumar A, Malik AK, Rai PK. SPME-HPLC: A new approach to the analysis of explosives. J Hazard Mat 2007;147;691-697.

17. Jiménez AM, Navas MJ. Chemiluminescence detection systems for the analysis of explosives. J Hazard Mat 2004;106A:1-8.

18. Nadezhdinskii AD, Ponurovskii YaYa, Stavrovskii DB. Non-contact detection of explosives by means of a tunable diode laser spectroscopy. Appl Phys B 2008;90:361-364.

19. Kolla P. Gas chromatography, liquid chromatography and ion chromatography adapted to the trace analysis of explosives. J Chromatogr A 1994;674:309-318.

20. Lagesson-Andrasko L, Lagesson V, Andrasko J. The use of gas-phase UV spectra in the 168-330-nm wavelength region for analytical purposes. 1. Qualitative measurements. Anal Chem 1998;70:819826.

21. Lagesson V, Lagesson-Andrasko L, Andrasko J, Baco F. Identification of compounds and specific functional groups in the wavelength region $168-330 \mathrm{~nm}$ using gas chromatography with UV detection. J Chromatogr A 2000;867:187-206.

22. Schug, KA Savicki I, Carlton Jr. DD, Fan H, McNair HM, Nimmo JP, Kroll P, Smuts J, Walsh P, Harrison D. Vacuum ultraviolet detector for gas chromatography. Anal Chem 2014;86:8329-8335. 23. Andrasko J. A simple microthermal desorption device. J Forens Sci 2006; 51:925-928. 
24. Tsyshevsky RV, Sharia O, Kuklja MM. Thermal decomposition mechanisms of nitroesters: Ab initio modeling of pentaerythritol tetranitrate. J Phys Chem C 2013;117:18144-18153. 


\section{Figure legends}

FIG. 1- UV spectrum of 2,4-DNT and TNT obtained on the GC-UV instrument. The temperature of the transfer line was $240^{\circ} \mathrm{C}$.

FIG. 2- A part of the three-dimensional chromatogram observed in the analysis of explosive mixture. The chromatogram shows the separation of EGDN and NG and the UV spectra of these compounds. Also dinitroglycerine is detected. The temperature of the transfer line was $150^{\circ} \mathrm{C}$. No decomposition to NO is observed at this temperature.

FIG. 3- UV spectrum for EGDN and NG extracted from the analysis presented in Fig. 2.

FIG. 4- A part of the three-dimensional chromatogram observed in the analysis of explosive mixture. The temperature of the transfer line was $220^{\circ} \mathrm{C}$. The diagram shows spectrum of NG and DNG which both decompose to NO at this temperature.

FIG. 5- The UV spectrum for NG detected at four different temperatures of the transfer line: $150^{\circ} \mathrm{C}$, $170^{\circ} \mathrm{C}, 200^{\circ} \mathrm{C}, 240^{\circ} \mathrm{C}$ (upper part). At higher temperatures NG decomposes to NO. The lower part of this figure shows the spectrum for NO obtained under the same experimental conditions.

FIG. 6- A three dimensional chromatogram obtained by microthermal desorption of TATP. The temperature of the transfer line was $180^{\circ} \mathrm{C}$. The peaks for air and free acetone are also detected. TATP decomposes partially to acetone which is co-eluted with the TATP peak. The UV-spectrum of pure acetone is presented in Fig. 7. The spectrum of acetone can be subtracted from that of TATP to find the UV spectrum of pure TATP. 
FIG. 7- UV spectrum of TATP detected at various temperatures of the transfer line $\left(160^{\circ} \mathrm{C}, 200^{\circ} \mathrm{C}\right.$, $220^{\circ} \mathrm{C}, 240^{\circ} \mathrm{C}$ ). The decomposition to acetone is enhanced at higher temperatures. UV spectrum of pure acetone is depicted in the lower part of this figure.

FIG. 8- UV spectrum of PETN recorded at the transfer line temperature of $180^{\circ} \mathrm{C}$ (upper part) and of pure PETN after subtraction of NO (lower part). PETN decomposes partially to NO in a manner similar to that of other nitrate esters. 
TABLE 1-Names, abbreviations and chemical structures of the explosives analyzed by GC-UV in this study.

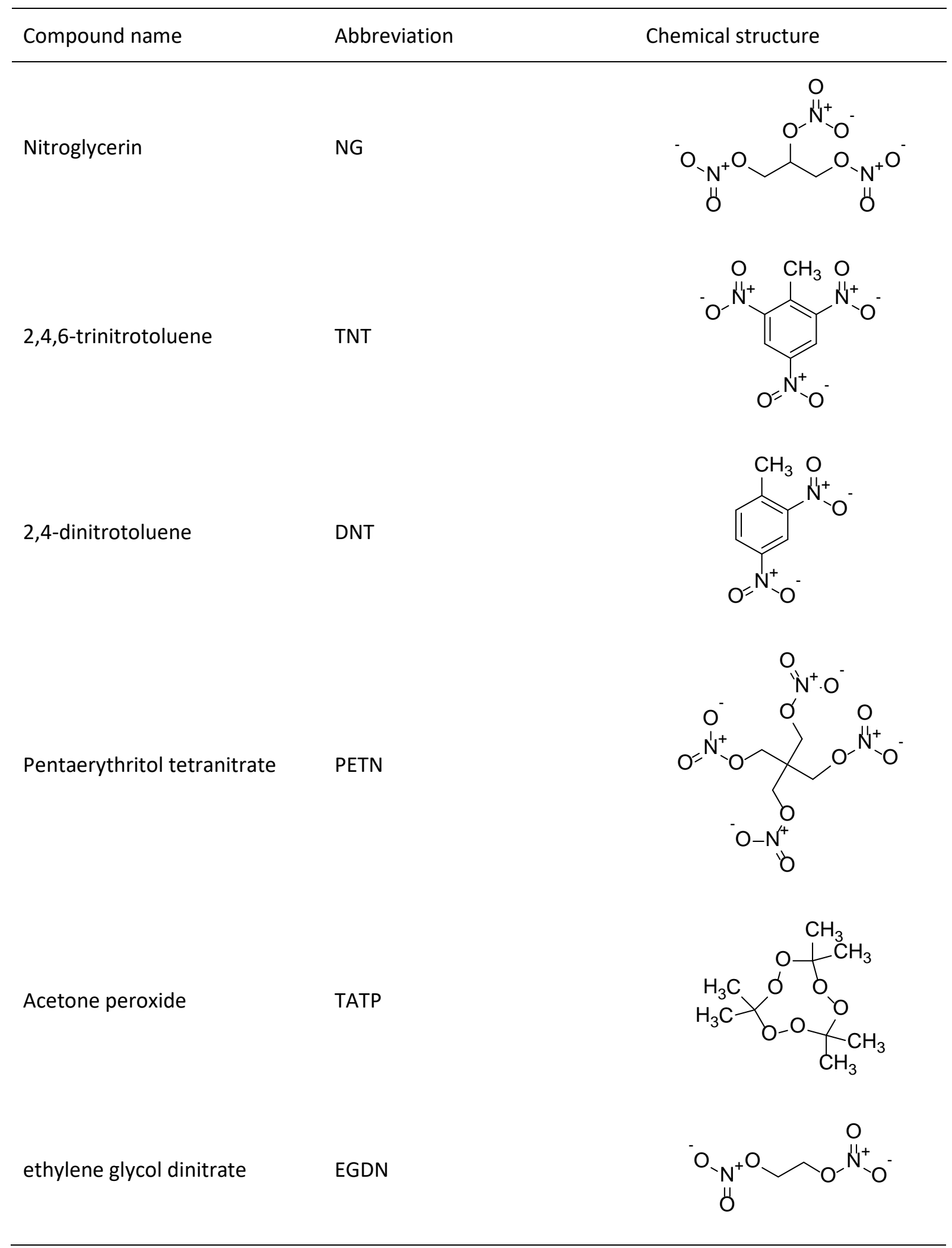


Fig. 1
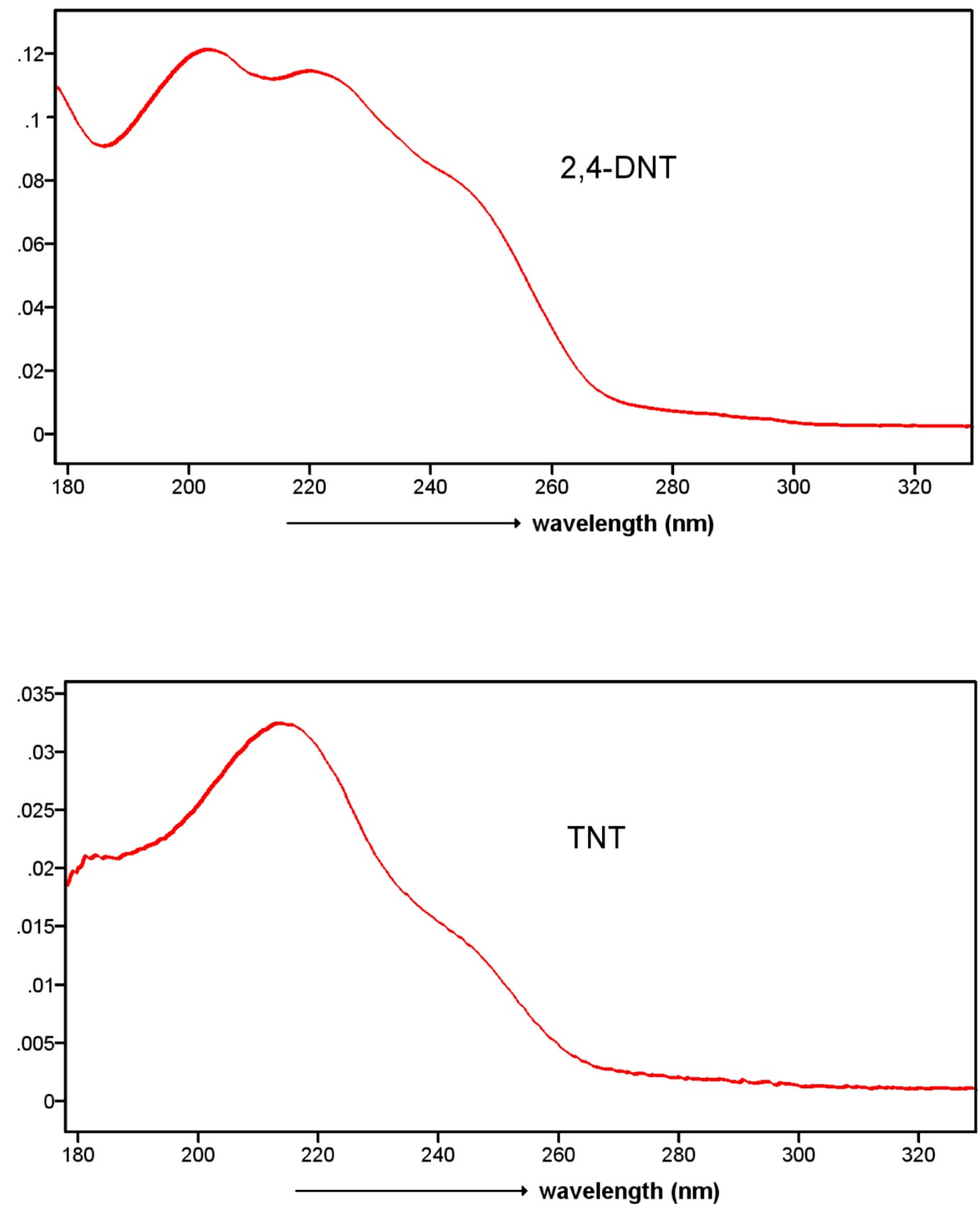
Fig. 2

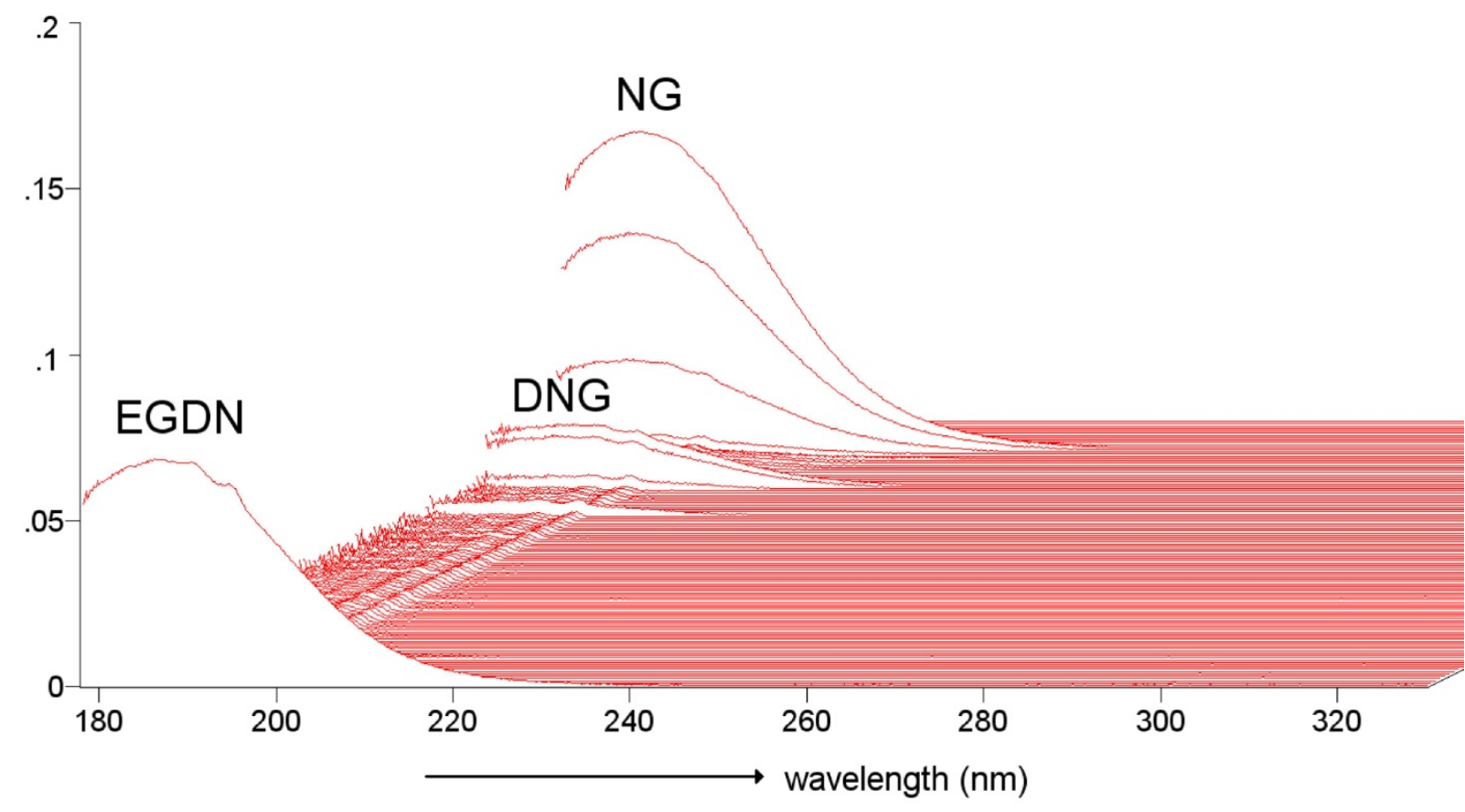


Fig. 3
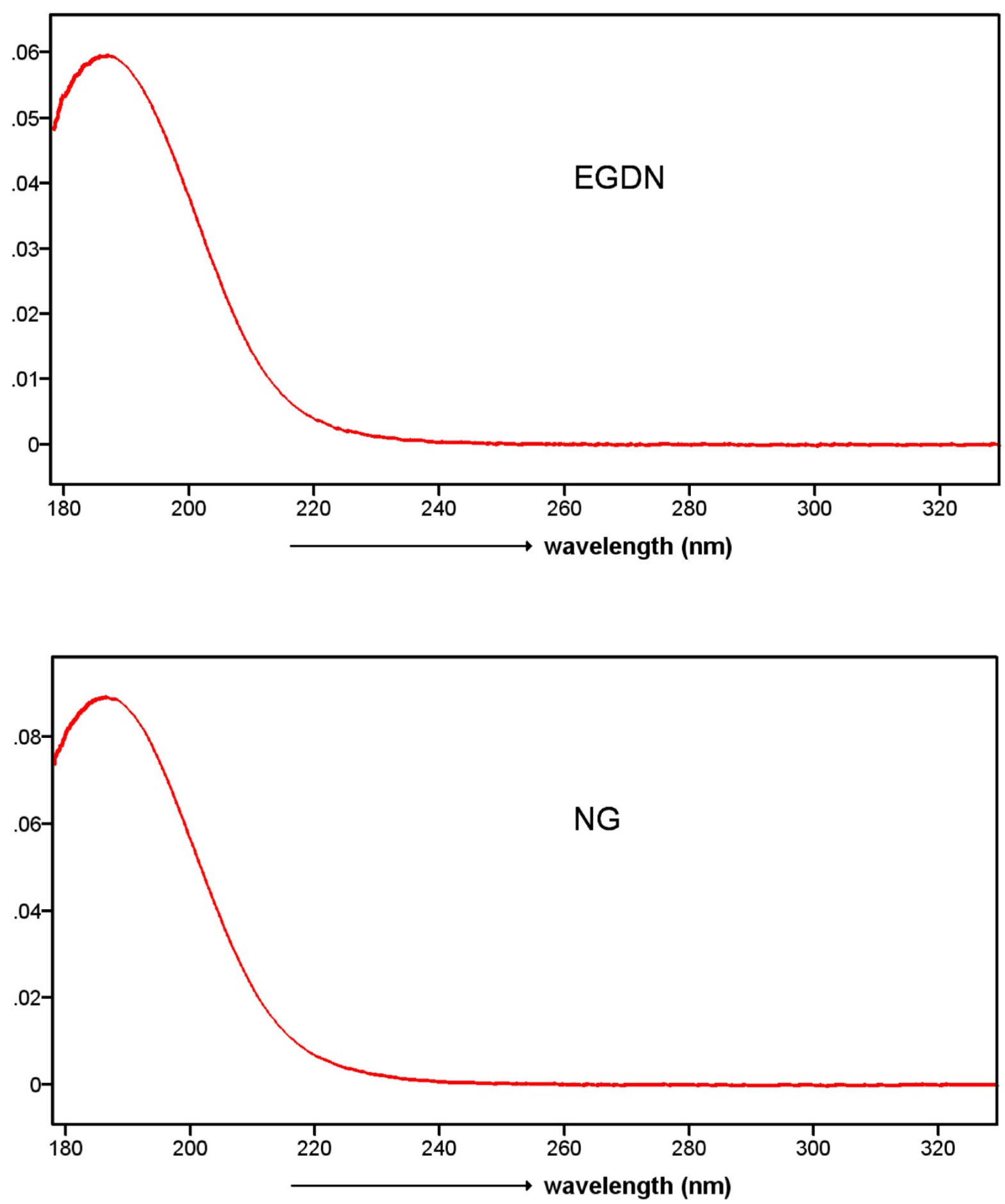
Fig. 4

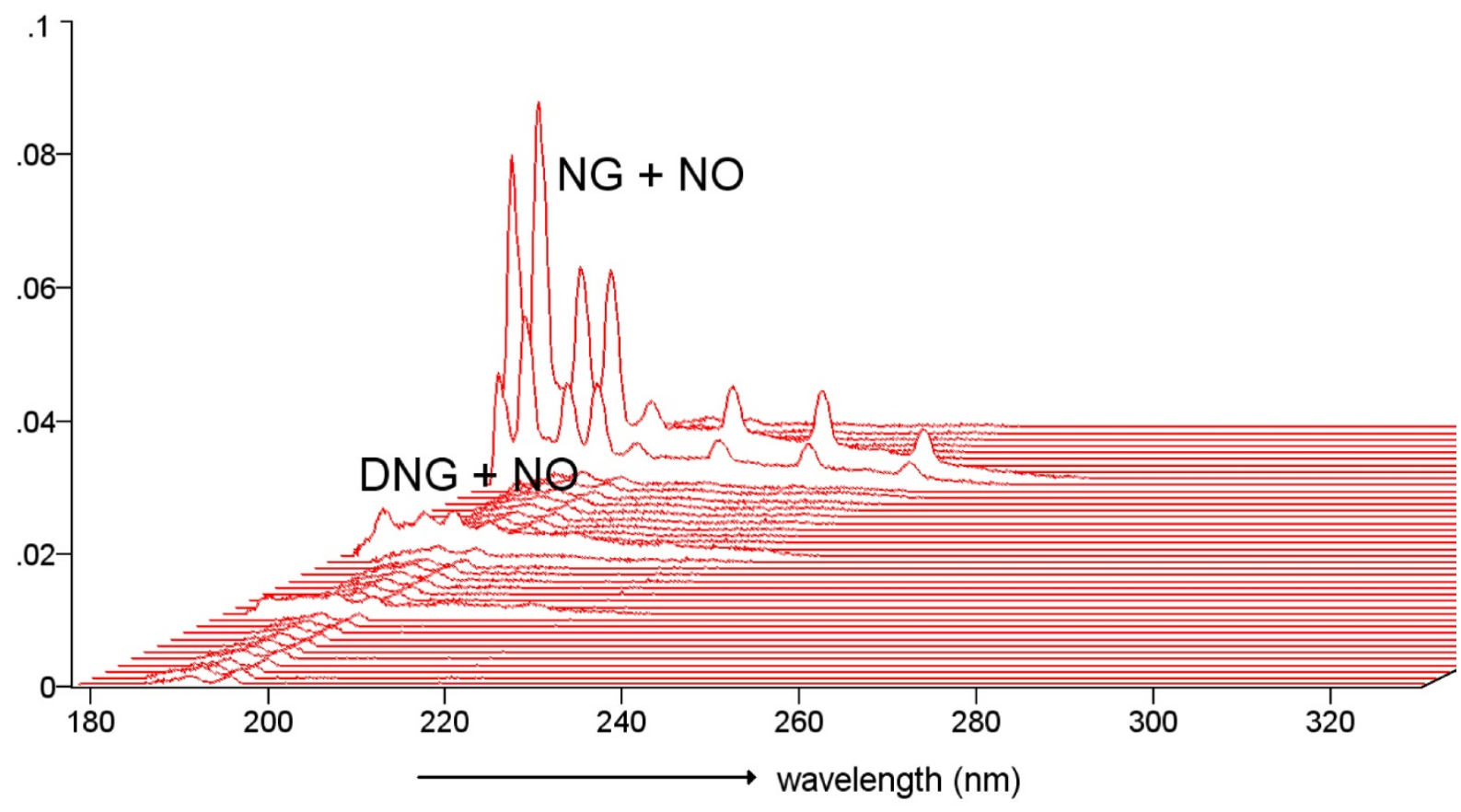


Fig. 5
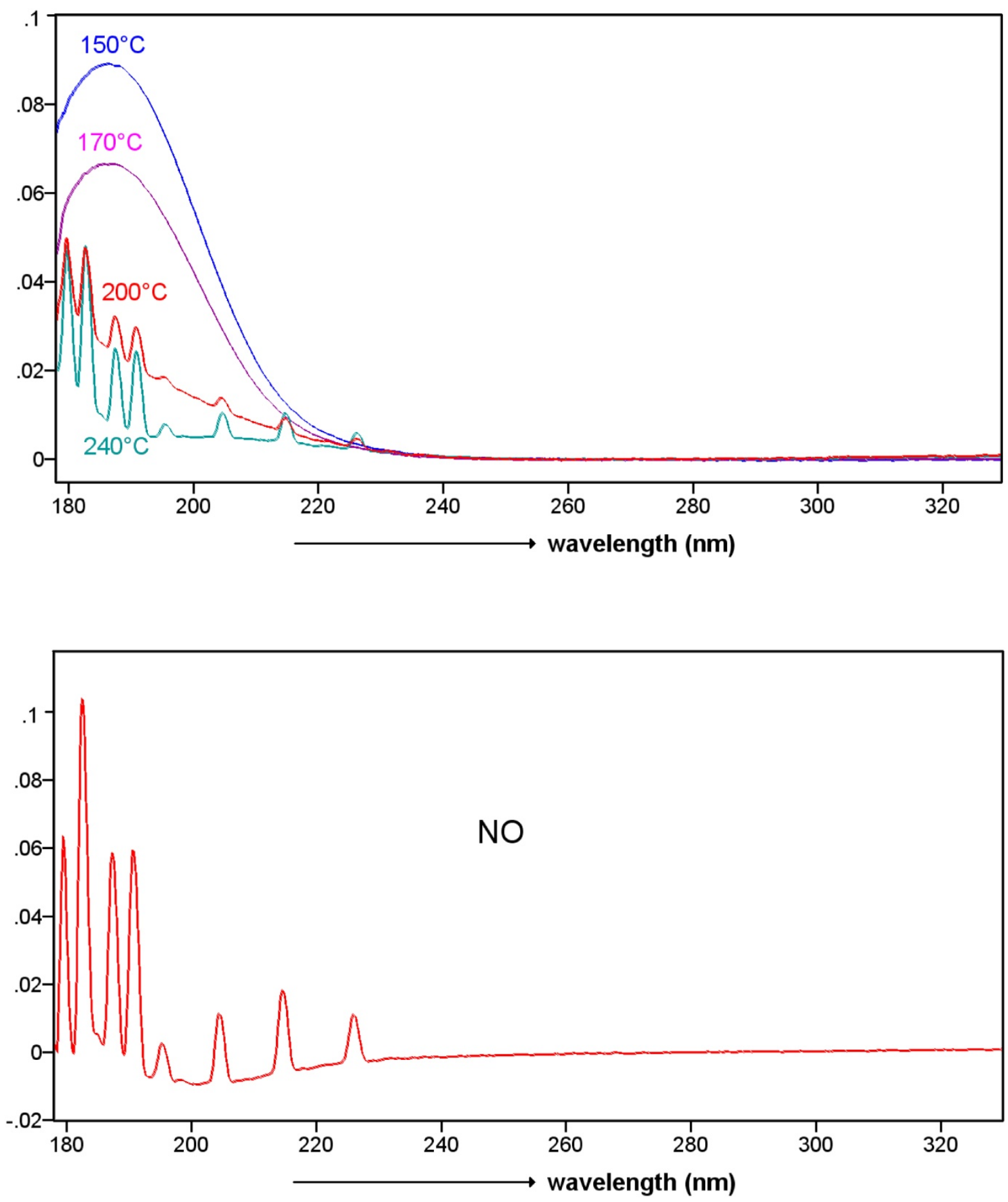
Fig. 6

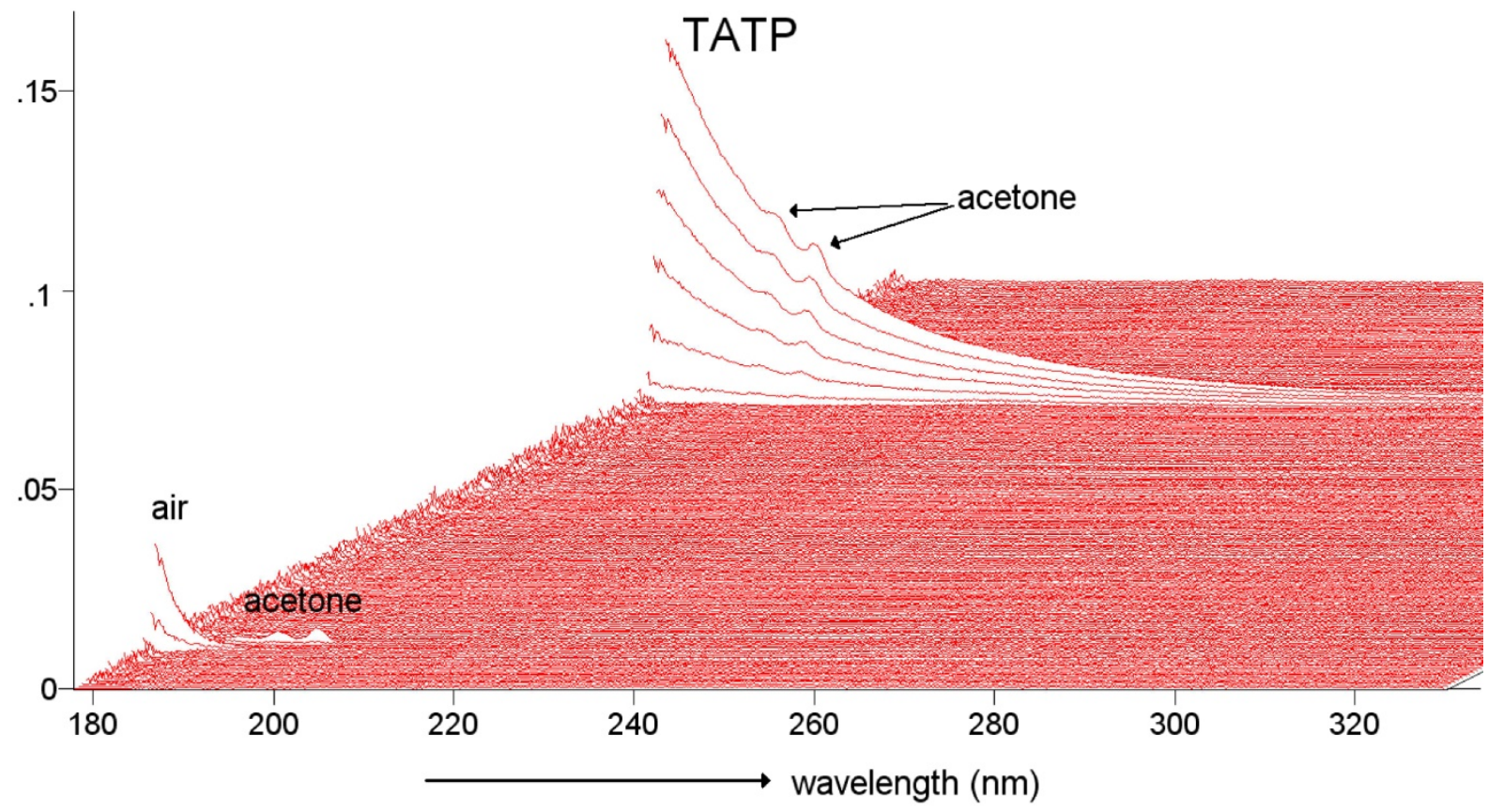


Fig. 7
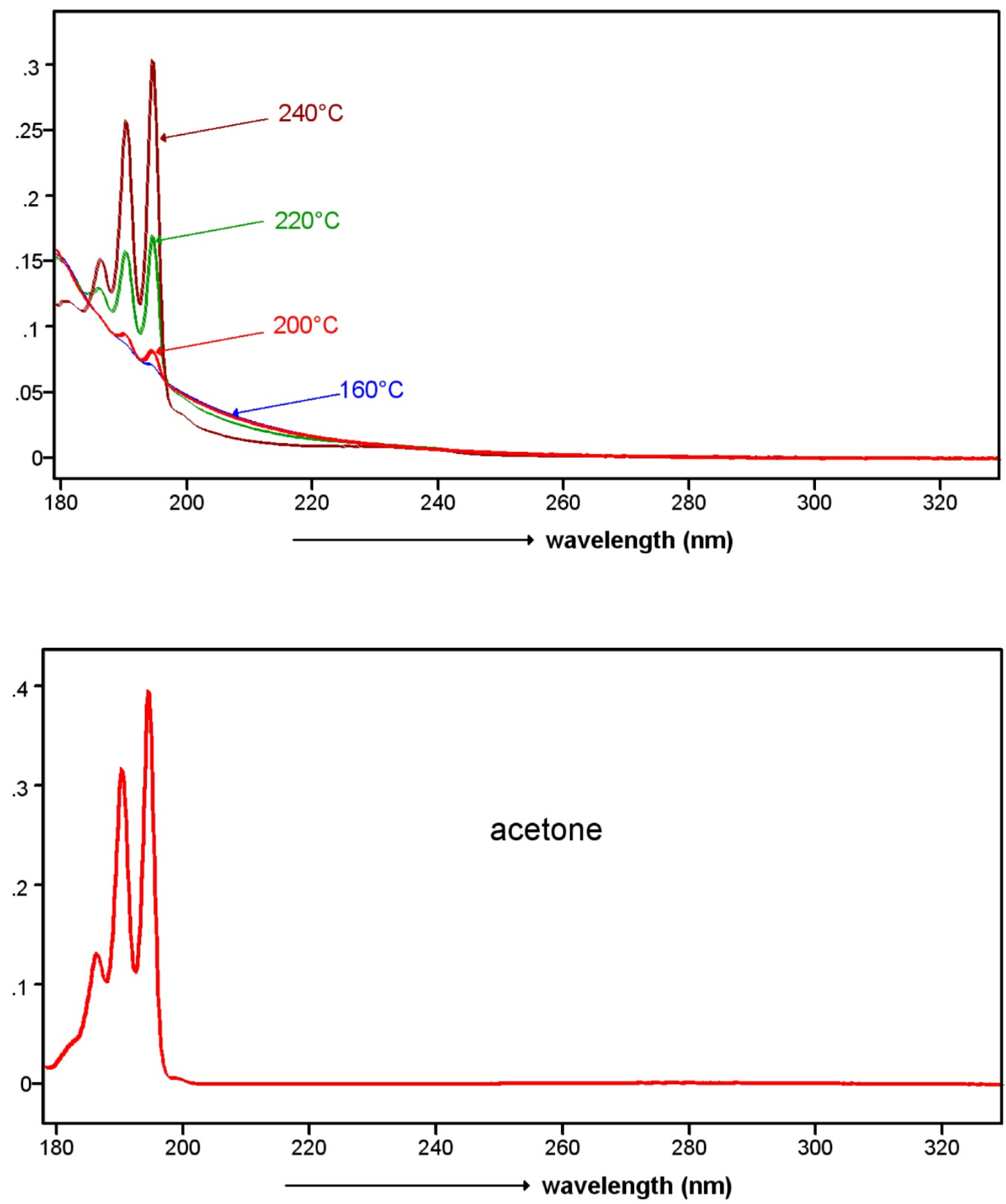
Fig. 8
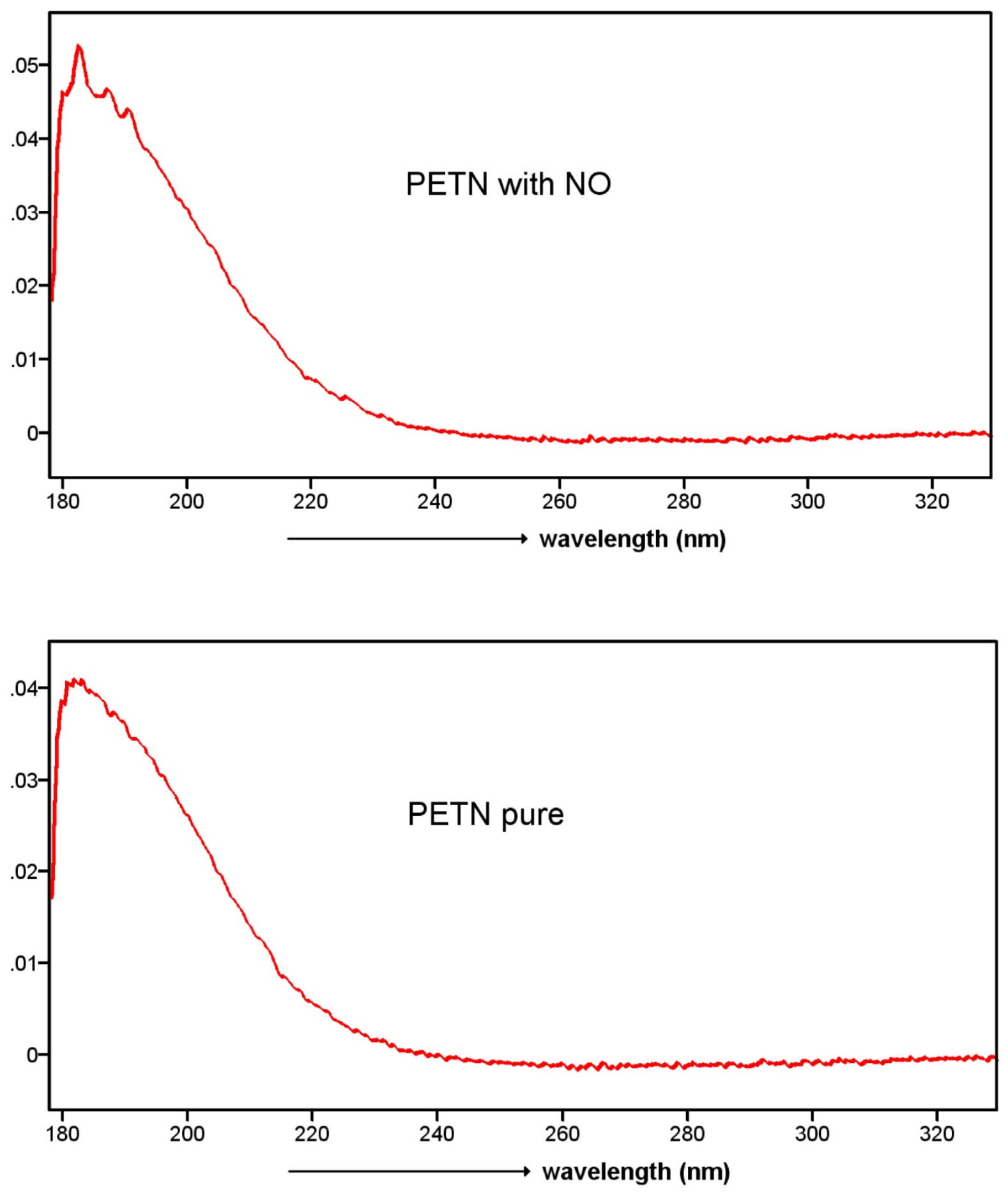